\title{
Integrating Feedback into Wearable Controls
}

\author{
Cátia Sousa and Ian Oakley \\ Madeira Interactive Technologies Institute, \\ University of Madeira, Caminho da Penteada, Funchal, Portugal \\ csfs.ferreira@gmail.com, ian@uma.pt
}

\begin{abstract}
Wearable computing is a highly specialized application domain requiring the development of novel interaction technologies. This is due not only to the distracted and eyes busy scenarios that such systems target, but also due to the necessity of implementing highly wearable systems that take advantage of the affordances of cloth and clothing. This paper presents the design and development of three novel wearable input devices based on commonplace elements of clothing: zippers, cords strung with beads and fabric patches. These devices implement different forms of input (respectively, linear continuous, linear discrete and tagging) and are notable in that they combine input with output in the form of persistent physical feedback - each device is designed to physically and visually resemble the digital information it controls. This paper argues that this approach is novel in the domain of wearable computing and has the potential to significantly improve usability.
\end{abstract}

Keywords: Smart textiles, wearable technology, craft materials, sensors.

\section{Introduction}

Integrating computation into human clothing is an intrinsically appealing idea. Wearable computers can aspire to be as ubiquitous as other practical tools built into our clothing (such as pockets) while providing access to sophisticated digital services, remaining continuously available for use and avoiding the burden of encumbering users with a discrete device such as a mobile phone. Careful design can fit wearable computers to our bodies for hands-free and eyes-free interaction in situations such as medical or sporting scenarios [1] and via emerging paradigms such as gestural interaction [2]. The flexible nature of cloth and clothing also affords novel opportunities for input, such as pulling or stretching cord-like appendages [3] or pinching different volumes of fabric [4]. These recent efforts to develop novel interaction techniques highlight the importance of designing wearable technologies and sensors that leverage the natural properties of fabric and clothing.

The work in this paper addresses this space. Its contribution is the design and implementation of three novel input techniques for wearable computers based on typical elements of clothing: a zipper, a bead on a cord and a system of removable fabric patches. These techniques are notable in that they each enable a different quality of input (respectively, linear continuous, linear discrete and tagging or identification) and are combined with persistent and visible physical alterations to clothing. The zipper is moved to continuously adjust a variable, the bead threaded 

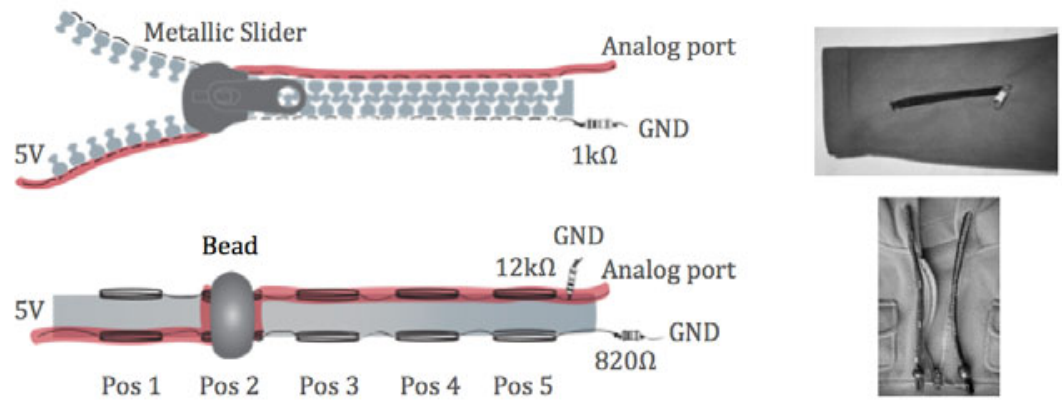

Fig. 1. Schematics for Zip and Toggle sensors (left). Sensors integrated into clothing (right).

along the cord to select specific modes and the patches added or removed to signify their presence or absence. Due to the complexity of embedding displays in cloth, such persistent feedback and state information is a rarity in wearable input techniques and this paper argues that its combination of rich input (when contrasted to commonly available binary pushbuttons [5]) coupled with visible feedback and output is a compelling new design space for research in interaction techniques for wearable computing. The introduction of this kind of coupled input/output will not only make wearable devices easier to use, but also offer designers and users new opportunities for making social and aesthetic statements.

\section{Zip and Toggle Sensors}

The first and second prototypes, based respectively on a zipper and a toggle (a cord strung with a bead) functioned as simple variable resistors. The zipper sensor was designed for continuous input while the toggle sensor allowed discrete, stepped input. Both devices incorporated two seams of 117/17 2-ply conductive thread, one sewn into each side. In both sensors, one of these lines connected a $5 \mathrm{~V}$ signal to ground (via an appropriate resistor), while the other was wired to an input port on an Arduino microcontroller. In the zip sensor, the conductive thread was strung along the surface of the zip material for its entire length, while the cord sensor featured a design in which the thread was alternately exposed and hidden beneath the non-conductive material of the cord at intervals of approximately one centimeter. The devices also integrated a conductive movable element in contact with both lines of thread: the metal slider of the zip and the cord's bead, the inner surface of which had been lined with copper tape. By adjusting the position of these physical devices, the length of thread making up the circuit, and thereby its resistance and the voltage delivered to the Arduino's input port, varied systemically and measurably. Pictorials schematics for these devices, and examples sewn into clothing, can be seen in Figure 1.

These devices have a number of interesting properties. They are both familiar to users, have minimal costs and are highly wearable - the main component is two seams of conductive thread. They also intrinsically provide two forms of feedback: the physical texture felt when adjusting the moveable element and its persistent, visually 
A
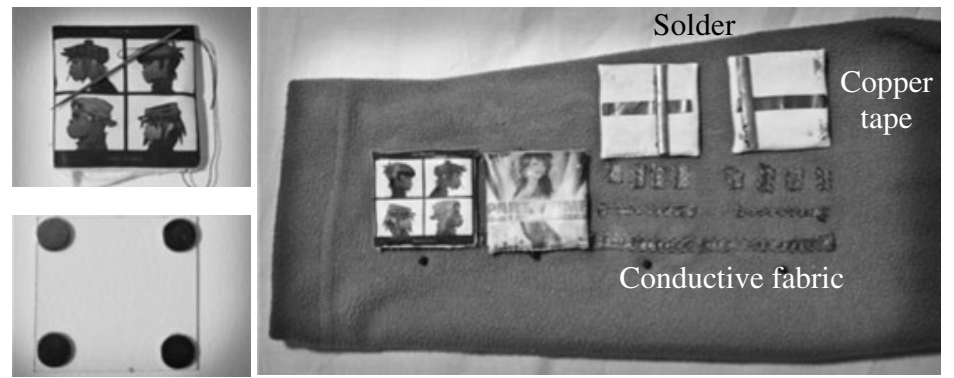

Fig. 2. Patch sensor A) front of final tag B) inner structure of tag showing rare-earth magnets at each corner $\mathrm{C}$ ) jacket sleeve with conductive fabric; two tags are attached and two tags show circuitry on the back of the tags

observable position. The physical state of the device mirrors the state of the sensed digital data. They can also both be integrated into the functional fastenings of an item of clothing to measure aspects of the garment state - for instance whether or not a jacket or pocket is open or closed. Alternatively, they can be sewn into a nonfunctional zone to serve as a control device capable of manipulating a variable such as the volume in wearable media player (continuous input with the zipper) or the ring tone profile selected on a mobile phone (discrete input with the cord and bead). We believe these devices provide general-purpose functionality for wearable applications.

\section{Patch Sensor}

The third sensor was based on a series of small tokens, or patches, which could be added to or removed from a piece of clothing capable of both sensing their presence and distinguishing between them. It employed several technologies to achieve this functionality. At the most basic level, the tokens were built from $45 \mathrm{~mm}$ by $45 \mathrm{~mm}$ squares of $4 \mathrm{~mm}$ Perspex. Small rare-earth magnets were attached to each corner of one side (the bottom) and the combined units encased in an outer layer of cloth. The surface of the bottom side was then augmented with a specific pattern of solder and copper tape while the top was used for distinctive graphical logos. Figure 2 shows a stripped down token and also examples of the top and bottom of complete units.

Figure 2 also shows the design of the apparatus that can sense and identify the tokens. It shows four "slots" in which tokens can be placed, two of which are occupied. Each slot features three rows of conductive fabric to which the patterns on the tokens connect. The bottom row is a $5 \mathrm{~V}$ power rail shared by all the slots. The center row of each slot connects to a different Arduino input port and voltage on these channels is used to indicate whether a token is present or absent. The top row featuring the four smallest fabric electrodes is used to identify individual tokens each also connects to a unique Arduino port. By connecting to different configurations of these electrodes 16 different binary patterns can be produced. Magnets positioned under the surface of the fabric ensure correct positioning and firm attachment of the tokens to the sensor unit. 
Although this device has significant limitations, not least in the limited number of tokens it can support (up to fifteen different tags), it does provide interesting functionality. The tokens attach firmly to the clothing, but are easy to add and remove; the sensor (and tokens) are composed of simple, flexible, lightweight and washable components; the use of multiple slots supports ordering; the tokens can be associated with visual content (such as the musical content shown in Figure 2) to match their digital identities. This final quality is particularly interesting as the visual elements not only provide information about system state, but can also be used to represent aspects of a user's wearable computer in a public form - perhaps as statements about brand, fashion or identity.

\section{Discussion and Conclusions}

This paper has presented three novel wearable sensors: linear continuous input in a zip, discrete continuous input in a cord and bead and object identification via a series of removal patches. The range of devices described achieves one of the key goals of this work: to explore input of different qualities and expressiveness. The second focus was on how wearable input could be made visible and persistent to human users and observers. This has also been amply achieved: each of the three input devices directly senses information that is equally visible to a human - each device looks like the variable it represents. The sensors are also inexpensive, wearable and designed to integrate well into clothing by adapting existing accessories and styles.

There are many avenues for future work on this topic, including: functional evaluations of the robustness of the input mechanisms to use in real clothing; improving and developing the feedback present in the devices, for example by integrating visual designs on the clothing, or by incorporating physical cues; user studies capturing both qualitative opinions and quantitative performance with the devices. In conclusion, three novel wearable sensors that look like the data they represent (and control), were presented. This paper argues that such feedback and transparency is an incongruous rarity in wearable input and that the prototypes effectively illustrate how it can be achieved. In this way, they point the way for further work exploring this topic.

\section{References}

1. Bächlin, M., Förster, K., Tröster, G.: SwimMaster: a wearable assistant for swimmer. In: Proceedings of Ubicomp 2009, Orlando, pp. 215-224 (2009)

2. Rekimoto, J.: GestureWrist and GesturePad: unobtrusive wearable interaction devices. In: Proceedings of the Fifth International Symposium on Wearable Computers, Zurich, pp. 2127 (2002)

3. Schwarz, J., Harrison, C., Hudson, S., Mankoff, J.: Cord input: an intuitive, high-accuracy, multi-degree-of-freedom input method for mobile devices. In: CHI 2010, pp. 1657-1660. ACM, New York (2010)

4. Karrer, T., Wittenhagen, M., Heller, F., Borchers, J.: Pinstripe: eyes-free continuous input anywhere on interactive clothing. In: Adjunct Proceedings of UIST 2010, pp. 429-430. ACM, New York (2010)

5. Fibretronic Linear Keypad, http: / /www. fibretronic.com/connectedwear/KP25SH 